\title{
STUDY ON THE APPLICATION OF AIRBORNE LIDAR IN SEISMIC ACTIVE FAULTS IN THE NORTHERN RIM OF QINLING MOUNTAIN AND THE PIEDMONT OF HUASHAN IN CHINA
}

\author{
Gaobo $\mathrm{He}^{*}$, Anni Wang \\ Aerial Photogrammetry and Remote Sensing Group Co., Ltd, Shaanxi, China - (505518576, 2825365661) @ qq.com
}

KEY WORDS: Airborne LiDAR, High-precision DEM, Active fault, Microtopography, Data preprocessing, Data post-processing

\begin{abstract}
:
Seismic active faults are closely related to earthquake disasters. Active faults determine the location and magnitude of destructive earthquakes. Therefore, strengthening active fault investigation and research is one of the main ways to reduce earthquake disasters. Airborne LiDAR, as a measurement method that can quickly obtain large-area and high-precision DEM results, can not only obtain structural distribution features of large-scale study areas on a macro scale, and determine the location and trend of active faults, but also be used to identify microstructural landforms and take researches on fine geomorphic structural features. In this paper, based on the detailed introduction, analysis and summary of the technical schemes, data processing and application of the airborne LiDAR technique in the northern margin of the Qinling Mountains and the Huashan Piedmont Fault Zone, it was demonstrated on the basic methods and technical advantages in seismic active faults and geomorphological research work of the airborne LiDAR technology, and a new and efficient method for seismic fault study was provided.
\end{abstract}

\section{INTRODUCTION}

The structure of the earth's crust rock layer that breaks due to the force reaching a certain strength, and the structure that has obvious relative movement along the fracture surface is called a fault. Active faults refer to faults that have been active since the Quaternary (or late Quaternary) and are still active. There are ample displacement evidences that can prove these faults have been active since 100,000 years (or 30,000 years) ago, or are active today, and are likely to remain active for some time in the future. Seismic active faults refer to active faults where earthquakes have occurred and may occur again. Active faults are closely related to the occurrences of the earthquake disasters. Active faults determine the location of most destructive earthquakes. Besides, the size of the active fault, the nature of the movement, and the age of the activity determine the magnitude of the earthquake magnitude. At the same time, they may have complicated impact on strong earthquake ground motion. Although we still cannot accurately predict the time, place and intensity of future earthquakes, as long as we know the exact locations of active fault distributions and their activity characteristics, we will know the places prone to earthquakes more easily. As a result, we can take economic and reasonable Earthquake Disaster Mitigation Measures. In a word, strengthening investigation and research on active faults is one of the main ways to reduce earthquake disasters.

Both the Qinling fault and the Huashan piedmont fault, which are located in the southern margin of the Weihe Basin in the Guanzhong region of Shaanxi Province, China, are earthquakeactive faults. Among them, the Huaxian $\left(34.5^{\circ} \mathrm{N}, 109.7^{\circ} \mathrm{E}\right)$ where the Huashan Piedmont fault was located, had a major earthquake around 24 o'clock on January 23, 1556, which was called "Guanzhong earthquake" in history. This earthquake is one of the most famous and historic earthquakes in China with the most extensive impact and the worst losses in one of the densely populated areas. Therefore, it is a necessary work for the seismic research department to carry out research on seismic faults in the northern margin of Qinling Mountains and the Piedmont of Huashan Mountain. However, due to the influence of large surface vegetation coverage and post-earthquake landform changes in the area, the discovery and identification of seismic faults in areas with an area of several hundred square kilometers has become a major obstacle affecting research work. How to efficiently obtain the high-precision DEM (Digital Elevation Model) necessary for active fracture research has been troubling seismic researchers, until the advent of airborne LiDAR measurement technology.

Due to its unique active non-contact measurement method and a certain penetration capability of the laser, the airborne LiDAR (Light Detection and Ranging) technique is less dependent on the weather. Compared with traditional photogrammetry technology, there is an evident advantage of high elevation accuracy as for the airborne LiDAR technique (She, 2018). The technique not only can quickly obtain a large area of highprecision DEM, but also can obtain the usable DEM of the region with vegetation coverage. The technique effectively solves the problem of obtaining real geomorphology and is of great benefit to the study of seismic faults.

In 2015, the airborne LiDAR work was implemented for further monitoring and researches for the Qinling fault and the Huashan piedmont fault.

\section{OVERVIEW OF THE STUDY AREA}

There were 4 main measurement objects in the northern margin of the Qinling Mountains and the Huashan Piedmont seismic active fault zone of the airborne LiDAR survey project: the northern Qinling fault, the Huashan Piedmont fault, the Weinan piedmont fault, and the Mazhao fault (Figure 1. A/B/C/H, Tian et al., 2003). The study area is divided into two discontinuous areas. The first area is from Yingtou Town of Meixian County,

\footnotetext{
* Corresponding author
} 
Jingzhou County, Huxian County, Chang'an District, and Tangying Town of Lantian County along the foot of the Qinling Mountains. The total length is about $152 \mathrm{~km}$ and the area is about $258 \mathrm{~km}^{2}$; Area 2 is from the suburb of Weinan City, Jinghua County to Maojiapo Village of Huayin County, with a total length of about $80 \mathrm{~km}$ and an area of about $107 \mathrm{~km}^{2}$.

The research goal was to obtain high-precision, high-density point cloud data and image data of about $365 \mathrm{~km}^{2}$ in the entire measurement area, and process the point cloud data and image data to obtain high-precision DEM and DOM data at 1: 1000 scale in the study area. The measurement area was striped along the northern foot of the Qinling Mountains. The narrowest part of the measurement area is about $1.2 \mathrm{~km}$ wide, the widest part is about $2.2 \mathrm{~km}$, and the average width is $1.4 \mathrm{~km}$. Because the measurement area is close to the mountain, the height difference within the measurement area is large and the maximum height difference exceeds $600 \mathrm{~m}$, which is unfavorable for aerial photography flight. The terrain in the survey area is complicated, and the ground control survey is difficult. The dense vegetation in the area is detrimental to accurately obtaining the DEM of the vegetation coverage area.

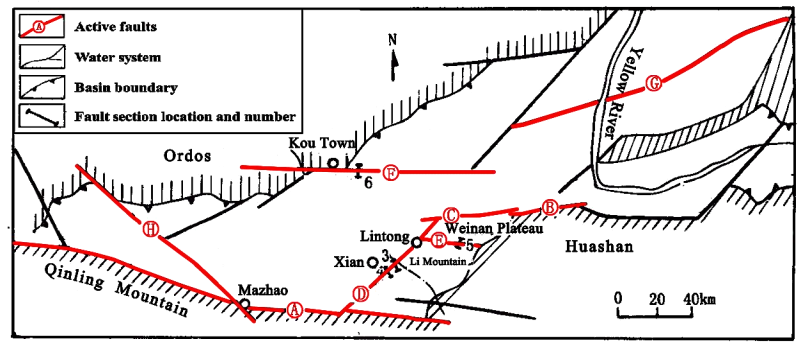

Figure 1. Schematic diagram of the location of major active faults in the Guanzhong area

(A: the northern Qinling fault; B: Huashan piedmont fault; C: Weinan piedmont fault; D: Lintong-Chang'an fault; E: Lishan piedmont fault; F: Kou Town-Guanshan fault; G: ShuangquanLintong fault; $\mathrm{H}$ : Mazhao fault)

\section{AIRBORNE LIDAR IMPLEMENTATION PLAN AND PRODUCTION ACQUISITION}

\subsection{Working Principle of Airborne LiDAR}

Airborne LiDAR integrates a number of high-tech technologies such as laser scanners, IMU inertial measurement units, differential GPS, and aviation flight control and management systems (Figure 2). Dependent on those equipment, the airborne LiDAR technique can achieve synchronous, fast and highly accurate acquisition of 3D coordinates and image data of ground targets (Dai et al., 2009; Shu, 2005). And real-time, realworld three-dimensional morphological characteristics of the reproduction can aabe quickly and intelligently realized. LiDAR's unique data acquisition method has made it apply in multiple fields, and it is one of the most revolutionary technological achievements in the field of photogrammetry and remote sensing in recent decades. Since 2003, LiDAR has gradually been adopted and recognized by the market as a mature high-tech technology. At present, in developed countries in Western countries, such as the United States, Germany and Asian countries, such as Japan, laser technology has been widely used, and its application fields include almost all aspects of economic construction, including seismic fracture research.

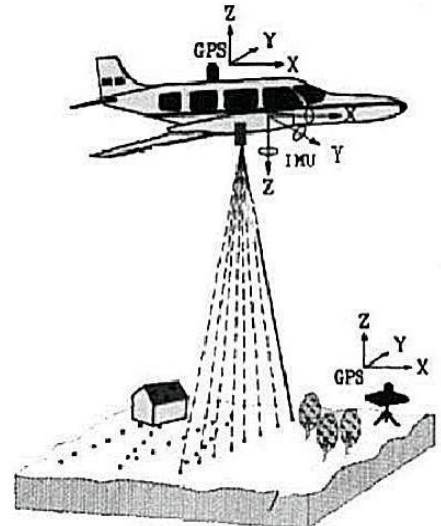

Figure 2. Schematic diagram of working principle of airborne LiDAR

The most basic working principle of LiDAR is no different from that of radio radar, that is, a signal is sent by the radar transmitting system, and is collected by the receiving system after the target reflects. And the distance of the target is determined by measuring the running time of the reflected light.

The distance measurement process mainly includes the following four steps.

(1) Laser emission

Under the action of the trigger pulse, the laser emits a narrow pulse, which is transmitted to the ground through the scanning mirror.

(2) Laser detection

The reflection from the ground target is collected through the scanning mirror. The returned echo signal is converted into an electrical signal by a photoelectric receiver.

(3) Time delay estimation

Processing the irregular echo signal accordingly, estimating the possible time delay of the target ranging, and giving the echo pulse signal. The delay of the pulse signal represents the delay of the target echo.

(4) Time delay measurement

The distance between the laser echo pulse and the laser emission pulse is measured by distance counting and other methods. Finally, the measured distance between the scanning ranging system and the ground target is obtained (Wan et al., 2007)

Namely (Ren et al., 2014):

$$
d=c * t / 2
$$

where $\quad d=$ distance from laser to reflecting object $c=$ speed of light $t=$ time.

The frequency of laser beam emission can be from several pulses per second to hundreds of thousands or even millions of pulses per second. The receiver will record dozens or even millions of points in one minute. Combining the laser position coordinate information obtained by GPS and the laser direction information obtained by INS, the geodetic coordinates X, Y, and $\mathrm{Z}$ of each laser point can be accurately calculated. A large 
number of laser points are aggregated into a laser point cloud to form dense point cloud data. Combined with the aerial images acquired synchronously, a variety of data results such as DSM / DEM / DOM / DLG can be obtained after data processing.

The airborne LIDAR system data is pre-processed to obtain 3D point cloud data, which has the following characteristics: (1) LIDAR data is discrete $3 \mathrm{D}$ point cloud data. One of the advantages of discrete data is that it allows the same plane coordinates to correspond to several elevations. Value, which is more conducive to the performance of detailed information (electrical lines in transmission lines, canopy structures, etc.) and rapidly changing terrain features (such as cliffs, buildings, etc.); (2) The laser point cloud has reflection intensity information, which reflects the response of the surface object to the laser signal; (3) Airborne Lidar point cloud data has the advantages of accurate elevation information and high vertical resolution compared to multispectral image data, but lacks the spectral and texture information of the image (Jiang, et al., 2007); (4) The spot density is different at different positions. The main reason for this is the different laser scanning methods.

\subsection{Airborne LiDAR Implementation}

In order to obtain more clear and accurate surface morphology in the study area, especially the micro-topography of key areas, the design requires that the density of ground point clouds obtained by LiDAR is not less than $10 \mathrm{pts} / \mathrm{m}^{2}$ (points / square meters). What's more, because the study area is on the edge of the Qinling Mountains with a large height difference, if a fixedwing aircraft is used as the flight platform, the flight height must be above $2200 \mathrm{~m}$ in order to ensure flight safety. The present LiDAR equipment could not perform laser scanning at this height to meet such a high point cloud density requirement at once. So it will take two or more repeated flights to meet the point cloud density requirement, which will inevitably increase the financial and time cost. After repeated research, we finally decided to use a helicopter as a flying platform. Due to the good air manoeuvrability of the helicopter, the flight height can be reduced to $500 \mathrm{~m}$ under the condition of ensuring safety. In addition, the selection of appropriate LiDAR equipment can ensure that sufficient point cloud density is obtained in one flight, thereby greatly reducing the flight time. At the same time, flying costs have been greatly reduced. After careful comparison and selection, we finally chose to take the Trimble Harrier 68i airborne LiDAR system (Figure 3) as the scanning device, and the American Bell Corporation 's 206 helicopter from the Bell Corporation of America (Figure 4) as the aerial photography flight platform.

The Harrier $68 \mathrm{i}$ produced by Trimble was one of the world's most powerful new-generation airborne $3 \mathrm{D}$ LiDAR systems at that time, and it was ahead of other similar products in terms of system stability, hardware performance indicators, and software support. The device has the ability to receive full waveform data with infinite waveform. And its maximum pulse frequency can be up to $400,000 \mathrm{~Hz}$, the distance accuracy can be up to $\pm 2 \mathrm{~cm}$, and it can be seamlessly combined with GPS, INS, digital cameras and other equipment. It can meet laser safety standards and allow safe operation at any altitude. The sampling frequency of the IMU inertial navigator is up to $200 \mathrm{~Hz}$. It is also equipped with the integrated high-resolution aviation digital camera with 60 million pixels features.

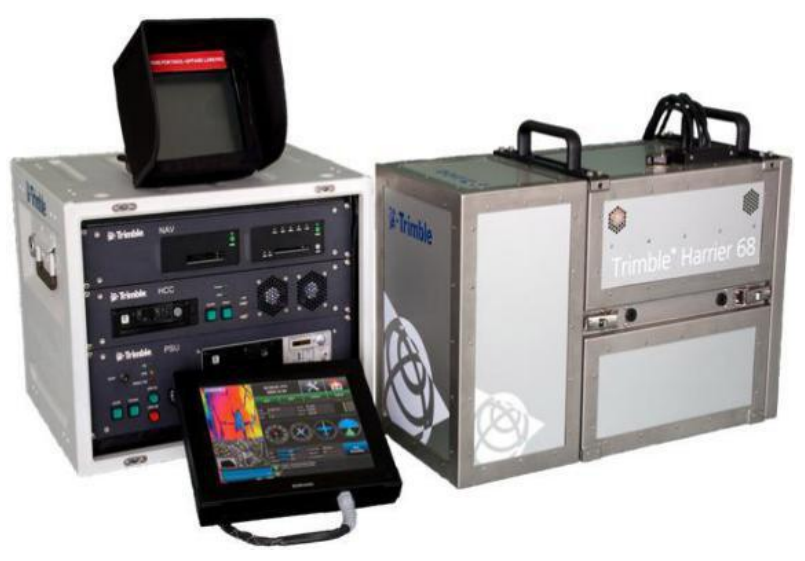

Figure 3. Harrier 68i LiDAR system

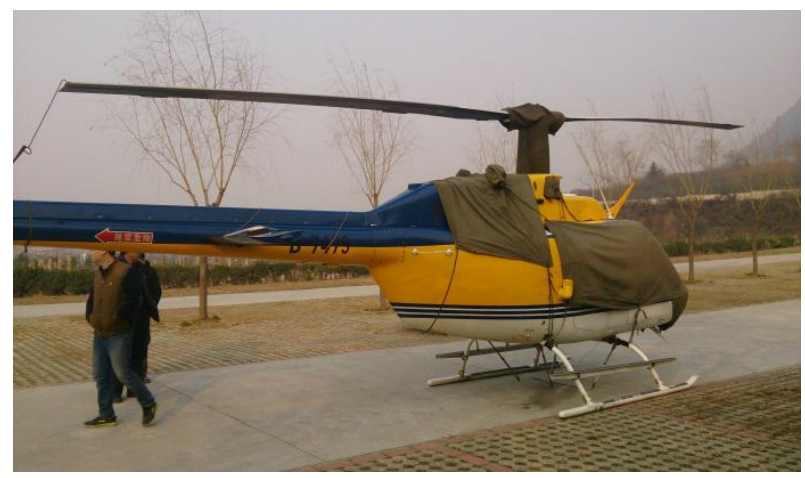

Figure 4. Bell 206 aircraft

And the final airborne LiDAR implementation plan and data processing flow were as showed in Figure 5.

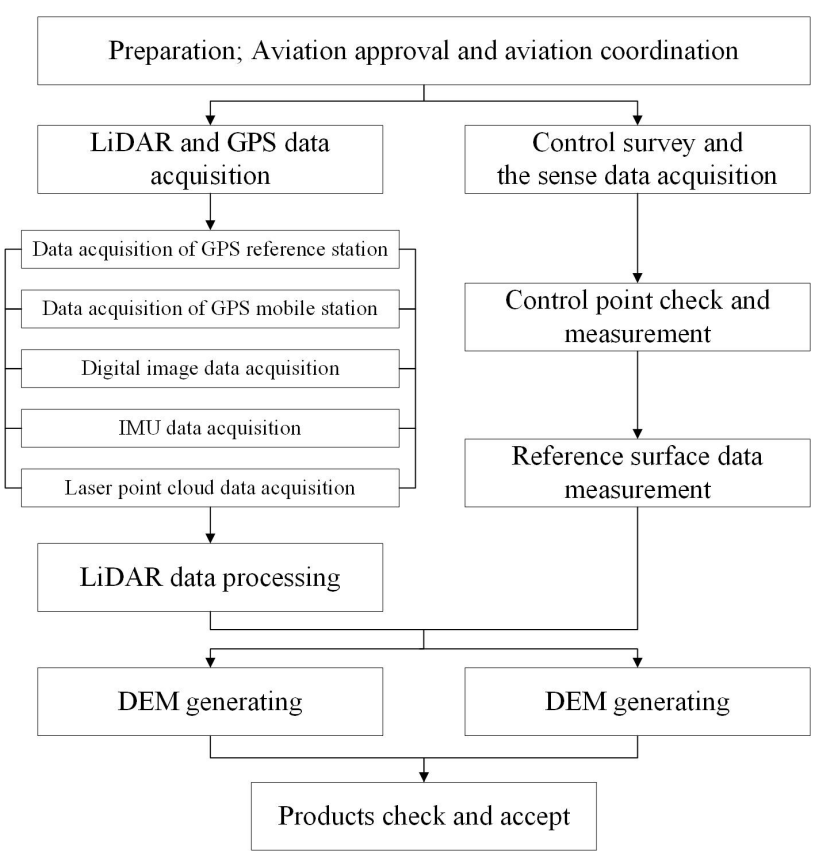

Figure 5. The flow chart LiDAR data processing

\subsection{Products Acquisition}

For the whole project, 9 aerial flights were implemented, 104 routes were completed, with the laser point cloud completely 
covering the entire survey area. A total of 8054 aerial photographs were taken, and the number of effective laser points obtained was about $6,275,041,208$. Airline overlap meets the given requirements without absolute loopholes; airline stitching meets the tolerance requirements.

\subsubsection{LiDAR and Raw Image Data Preprocessing}

There are two tasks to complete the data preprocessing: point cloud data solution and air strip correction.

\section{(1) Point cloud data solution}

The main work of the point cloud data solution is to decode the original data and obtain GPS data, IMU data, laser ranging data, etc. Sort the POS data, IMU data, flight record data, base station data, and laser ranging data of the same flight to generate point cloud data that meets the requirements. Combine the airborne POS data and the CORS station data to calculate the track, and import the solution software to solve the LIDAR data to obtain the laser point cloud data of each flight. The laser point cloud data of the entire measurement area is obtained through the flight zone matching and the elimination of system errors.

The density of the densest point cloud area was more than 65 pts $/ \mathrm{m}^{2}$, and the density of the sparsest point cloud area was greater than $11 \mathrm{pts} / \mathrm{m}^{2}$ (excluding some special areas such as water areas), and the average point cloud density is about 17.43 pts $/ \mathrm{m}^{2}$; after classification and processing of point clouds, the effective ground points obtained account for about $63 \%$ of the total number of point clouds, and the average ground point cloud density is about $10.98 \mathrm{pts} / \mathrm{m}^{2}$, completely better than the $10 \mathrm{pts} / \mathrm{m}^{2}$ required by the project design.

\section{(2) Air strip correction}

The original laser point cloud data obtained must took airline correction, that is, the original point cloud data was planarized according to the measured plane and elevation control points and elevation correction to calculate the spatial threedimensional coordinates of the surface object point cloud.

\subsubsection{LiDAR Data Post-processing}

A $500 \mathrm{~m} * 500 \mathrm{~m}$ block was used as the processing unit for all qualified aeronautical point cloud data, and the first echo data of the corrected 3D laser point cloud data was used for denoising and interpolation to produce a digital surface model (DSM, Figure 6) that reflects surface information

At the same time, the corrected 3D laser point cloud was used for classification to generate ground points and non-ground points, and then extract the point cloud data of the last echo based on the classified ground points, and then gridding (framing), filling small gaps, removing gross points, filtering and interpolation to make a digital elevation model (DEM, Figure 7).

Finally, based on the DEM and the obtained aerial image data, DOM were produced. And the DEM produced was combined with ground control point data to orthorectify the images, uniformize and stitch them. After image mosaic lines were made, framing processing was performed according to the mosaic line and the even-colored image to produce the final digital orthophoto model (DOM, Figure 8) map.

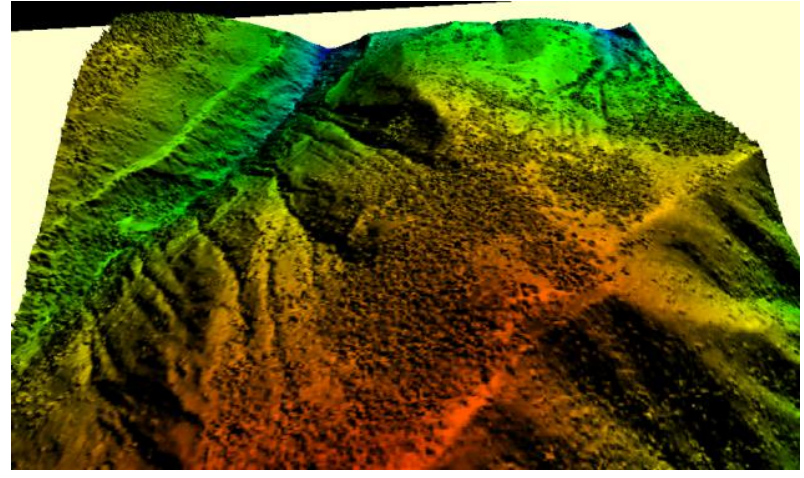

Figure 6. DSM product

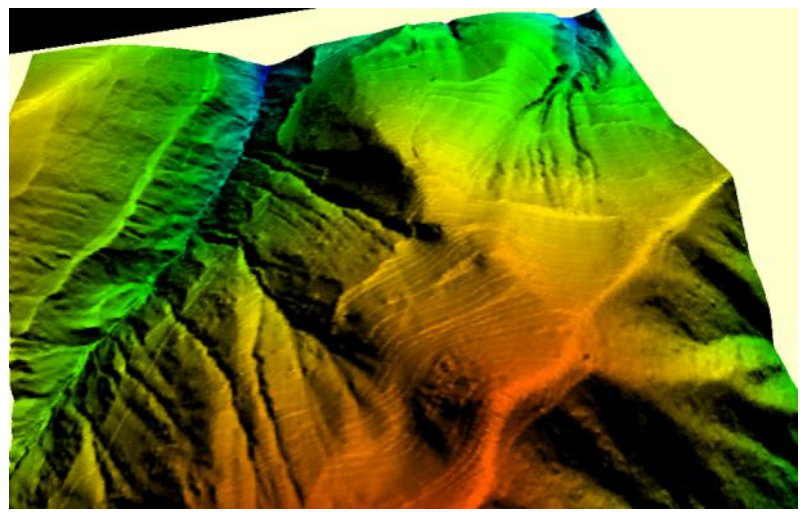

Figure 7. DEM product

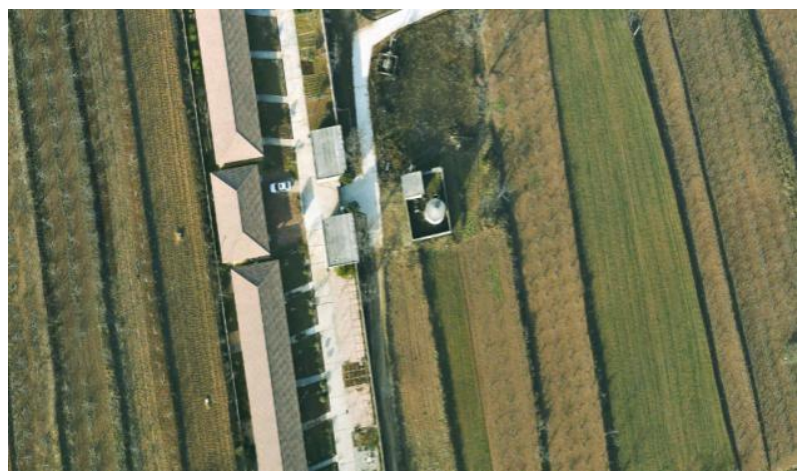

(a)

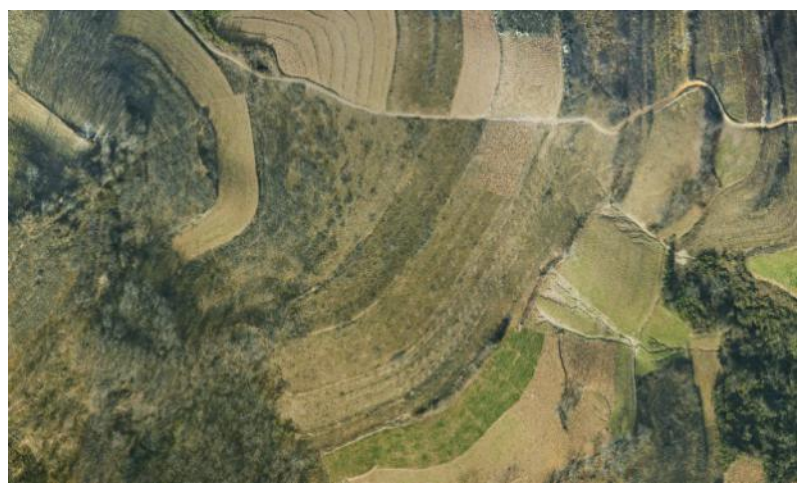

(b)

Figure 8. DOM product 


\subsubsection{Accuracy Test}

The field control point data was used to check the accuracy of the DEM product and DOM product of the research area. According to the standards (Table 1) in the Chinese file called Airborne LiDAR data processing technical specifications $(\mathrm{CH} T$ 8023-2011), the maximum error of the results (Table 2) was less than the standards of 1: 1000 topographic map specifications. So the accuracy met the requirements.

\begin{tabular}{|c|c|c|}
\hline $\begin{array}{c}\text { Terrain } \\
\text { category }\end{array}$ & DEM elevation error $/ \mathrm{m}$ & DOM plane error $/ \mathrm{m}$ \\
\hline Flat land & 0.2 & 0.6 \\
\hline Hilly land & 0.5 & 0.6 \\
\hline Mountain & 0.7 & 0.8 \\
\hline Alpine & 1.5 & 0.8 \\
\hline
\end{tabular}

Table 1. Accuracy requirements of 1: 1000 topographic map

\begin{tabular}{|c|c|c|}
\hline & DEM elevation error $/ \mathrm{m}$ & DOM plane error $/ \mathrm{m}$ \\
\hline Max & 0.18 & 0.26 \\
\hline Min & 0.05 & 0.09 \\
\hline
\end{tabular}

Table 2. Actual accuracy of the products

\subsection{Application of Products in Seismic Faults}

The results were mainly used in the discovery and identification of unknown faults, and the research and evaluation of the surface morphology and microtopography of known faults. And it turned out that the application effect was satisfactory.

For DEM results, based on the theories of quantitative geomorphology, digital terrain analysis method, morphological analysis method (Wei, et al., 2014; Liu, et al., 2016; Han, et al., 2017), et al., it was easy to find the exact location and direction of a fault in the front of the Huashan (Figure 9) through multiangle browsing and other operations, and also to measure its length, height, range, etc..

In addition, the DOM results were used as the texture of the terrain data, and helped to construct the three-dimensional interpretation environment in which the slope, aspect, vegetation coverage, altitude, rock formation and other information were more realistically showed.

Comprehensive consideration of the texture information, morphological information, and shape information of the fault zone can better provide reliable data for seismic fault researches (She, et al., 2018).
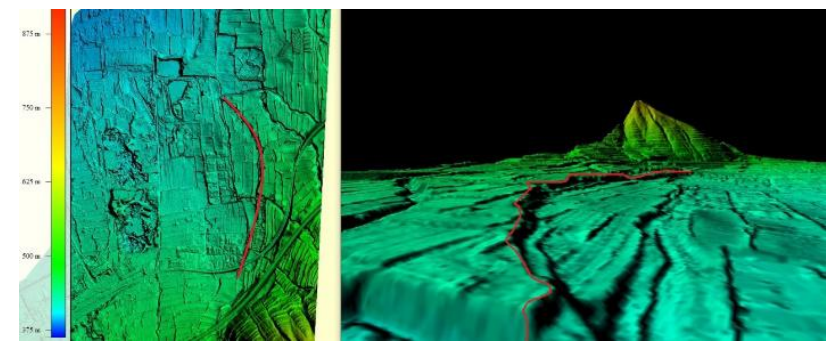

Figure 9. Location and three-dimensional view of a fault in front of Huashan Mountain

\section{CONCLUSIONS AND PROSPECTS}

At present, in the study of seismic faults in China, traditional aerial photogrammetry or ground surveying (GPS / RTK or total station) is still the main technical means to carry out fault and geomorphological-related surveys. Compared with traditional measurement technology, the airborne LiDAR technology has great technical advantages and comprehensive economic advantages, which are mainly reflected in the following aspects:

(1) The overall accuracy and fineness of the results are higher First of all, each point of the airborne LiDAR point cloud is directly measured by laser. But the elevation points of traditional aerial survey or ground survey are basically collected by the operators, which indicated great influence of human factors. Therefore, the overall accuracy of LiDAR point cloud is better. Secondly, the density of the original points collected by airborne LiDAR is also much higher than that of traditional aerial surveys or ground surveys. And the denser the point cloud is, more finely the terrain and landform can be expressed, which is beneficial to earthquake research. On average, the airborne LiDAR technique can produce dozens or even dozens of raw points per square meter, which is incomparable with traditional aerial survey collection or manual collection in the engineering survey. Furthermore, the accuracy of airborne LiDAR elevation measurement is higher than other surveying and mapping methods, especially in large vegetation coverage or other special areas that have greater difficulties working with traditional surveying methods. Because the LiDAR laser has a strong penetrating ability, it can obtain more accurate terrain surface data. The aerial photogrammetry method requires the operator to estimate the tree height before the terrain surface data can be obtained, so the measurement error, especially elevation accuracy is relatively large.

(2) Higher production efficiency and shorter project period The altitude of airborne LiDAR, especially helicopter-borne LiDAR, is relatively low. Also it actively emits laser pulses for measurement, so the flight is less affected by weather than traditional aerial surveys, which means that the flying days can be longer. In addition, the on-board LiDAR measurement technology requires only a small amount of manual field survey work. The office work is with a high level of intelligent and automated production. The production cycle is greatly shortened, for the reduce of the traditional aerial survey production link, such as field image control point measurement and Threedimensional triangulation. The 3D laser point cloud data can quickly and directly obtain products such as DSM / DEM, and can obtain a large area of DEM or DSM in a short period of time.

\section{REFERENCES}

Dai Yajing, Shen Xuhui, Hong Shunying, 2009. Research on airborne LiDAR and its application in earth science. Earthquake, 29 (supplement): 122-129.

Han Nana, Shan Xinjian, Song Xiaogang, 2017. VHR DEM measurement technology and its application in active fault research. Acta Seismologica Sinica, 39 (3): 436 - 450.

Jiang Jingjue, Zhang Zuxun, Ming Ying, 2007. Filtering of LiDAR point clouds for complex cityscape. Geomatics and Information Science of Wuhan University, 32 (5): 402-405.

Liu Yupin, Liang Hong, Chen Feifei, 2016. Application of high resolution airborne LiDAR in Xiaojiang active tectonics and geological disaster study. Journal of Geomechanics, 22 (3): 747 -759 . 
Ren Zhikun, Chen Tao, Zhang Huiping, et al., 2014. LiDAR survey in active tectonics studies: an introduction and overview. Acta Geologica Sinica, 88 (6): 1196-1207.

She Jinxing, Cheng Duoxiang, Liu Fei, et al., 2018. Application of airborne LiDAR technology in geological disaster investigation - taking the Jiuzhaigou MS7.0 earthquake in Sichuan Province as an example. Earthquake Research in China, 34 (3): 435-444.

Shu Ning, 2005: Laser Imaging. Wuhan University Press, Wuhan.

Tian Qinjian, Shen Xu hui, Feng Xijie, Wei Kaibo, 2003. Primary study on quaternary tectonic events based on variation of fault activity in Weihe basin. Seismology and Geology, 25 (1): 146-154.

Wan Youchuan, Xu Jingzhong, Lai Xudong, Zhang Shengwang, 2007. Filtering of LiDAR points clouds based on multiresolution directional prediction. Geomatics and Information Science of Wuhan University, 32 (11): 1011-1015.

Wei Zhanyu, He Honglin, Gao wei, et al, 2014. Experimental study on geologic mapping of active tectonics based on LiDAR data - a case of Dushanzi anticline - reverse fault zone in Xinjiang. Seismology and Geology, 36 (3): 794 - 813. 